\title{
BMJ Open Periconception endogenous and exogenous maternal sex steroid hormones and risk of asthma and allergy in offspring: protocol for a systematic review and meta-analysis
}

\author{
Merhunisa Talovic, ${ }^{1}$ Aziz Sheikh, ${ }^{1}$ Nicola McCleary, ${ }^{1}$ Maijaliisa Erkkola, ${ }^{2}$ \\ Minna Kaila, ${ }^{3}$ Suvi M Virtanen, ${ }^{4,5,6,7}$ Bright I Nwaru ${ }^{1,4}$
}

To cite: Talovic M,

Sheikh A, McCleary N, et al. Periconception endogenous and exogenous maternal sex steroid hormones and risk of asthma and allergy in offspring: protocol for a systematic review and meta-analysis. BMJ Open 2017;7:e014637. doi:10.1136/ bmjopen-2016-014637

- Prepublication history and additional material for this paper are available online. To view these files please visit the journal online (http://dx.doi. org/10.1136/bmjopen-2016014637).

Received 16 0ctober 2016 Revised 15 May 2017 Accepted 24 May 2017

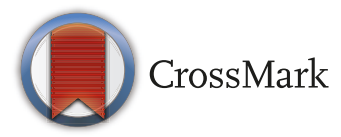

For numbered affiliations see end of article.

Correspondence to

Merhunisa Talovic;

merhunisa.talovic@gmail.com

\section{ABSTRACT}

Introduction Pregnancy is associated with several hormonal changes which influence the developing fetus. Variations in maternal endogenous hormones and prepregnancy use of hormonal preparations have been linked to asthma and allergy in the offspring, but findings are inconsistent. We plan to undertake a systematic review to synthesise the evidence on the association between endogenous and exogenous maternal sex hormones and the risk of asthma and allergy in the offspring.

Methods and analysis We will search Medline, Embase, Cochrane Library, Institute of Scientific Information Web of Science, Cumulative Index of Nursing and Allied Health, Scopus, Google Scholar, Allied and Complementary Medicine Database, Global Health, Psychological Information (PsycINF0), Centre for Agriculture and Bioscience (CAB) International and WHO Global Health Library from inception until 2016 to identify relevant studies on the topic. Additional studies will be identified by searching databases of proceedings of international conferences, contacting international experts in the field and searching the references cited in identified studies. We will include analytical epidemiological studies. Two researchers will independently screen identified studies, undertake data extraction and assess risk of bias in eligible studies, while a third reviewer will arbitrate any disagreement. We will use the Effective Public Health Practice Project tool to assess the risk of bias in the studies. We will perform a random-effects meta-analysis to synthesise the evidence. We will use the Grading of Recommendations Assessment, Development and Evaluation approach to rate the strength and quality of the overall evidence with respect to each outcome.

Ethics and dissemination Ethical approval is not required since the study is a systematic review of published literature. Our findings will be reported in a peer-reviewed scientific journal.

PROSPERO registration number CRD42016048324

\section{INTRODUCTION}

Pregnancy is associated with numerous adjustments that occur in the endocrine system, which are normal and necessary adaptations
Strengths and limitations of this study

- This is the first systematic review examining the role of endogenous and exogenous maternal sex steroid hormones and risk of asthma and allergy in the offspring and thus will contribute to knowledge on the fetal origin of allergy and asthma.

- We will identify studies from major healthcare databases without using geographical or language limitations, as well as including an extensive review on all of the current literature, ongoing and unpublished studies and will employ replicable methods.

- Existing studies may be heterogeneous, thus making it difficult to derive pooled estimates from included studies.

that adequately accommodate the fetus. ${ }^{1-5}$ These adjustments include oestrogen and progesterone hormonal changes, which see steady levels of increase and are essential in suppressing the hypothalamic axis and the menstrual cycle. ${ }^{1-5}$ Human chorionic gonadotropin also increases and helps to sustain the production of progesterone by the corpus luteum. ${ }^{45}$ Prolactin levels increase as a result of the maternal pituitary gland. Adrenal hormones such as cortisol and aldosterone increase, as do parathyroid hormones, which lead to greater uptake of calcium in the gut and reabsorption in the kidney. ${ }^{1-5}$

Oestrogen plays a key role in the well-being and development of the fetus. ${ }^{45}$ Increases in the levels of progesterone production help to relax bronchiolar smooth muscle and are associated with an increase in minute ventilation, which can rise to up to $50 \%$ greater than prepregnancy levels. ${ }^{45}$ Both oestrogen and progesterone are thought to act as immunosteroids, although the effect on the direction 
of shift in the T cells is not entirely clear. ${ }^{45}$ However, it has been suggested that the observed changes in levels of maternal endogenous sex steroid hormones, including oestrogen, progesterone and testosterone, during pregnancy may influence subsequent risk of asthma and allergy in the offspring. ${ }^{6-9}$

Prepregnancy use of oral contraceptive pills, including combined pills and progestin-only pills, has been postulated to modulate the risk of asthma and allergy in the offspring through possible disruption of the immunological balance. ${ }^{10-16}$ Wjst and Dold were the first to postulate that the use of contraceptive pills before pregnancy may influence subsequent risk of asthma and allergy in the offspring. ${ }^{10}$ According to their hypothesis, the effect of contraceptive pills may last for several years even after discontinuation; however, the dosage, frequency and timing of use of oral contraceptive pills which may instigate the immune effect that can result in increased offspring asthma and allergy risks are unclear. ${ }^{10}$

Although several studies have now investigated the role of both endogenous and exogenous sex steroid hormones during pregnancy in the development of asthma and allergy in the offspring, findings are inconsistent. To clarify the underlying evidence base, we plan to undertake a systematic review to identify, critically appraise and synthesise studies on the role of changes in endogenous sex steroid hormones during pregnancy and the role of exogenous steroid hormone use before pregnancy in the development of asthma and allergy in the offspring.

\section{METHODS}

This protocol is reported following the recommendations of the Preferred Reporting Items for Systematic review and Meta-Analysis Protocols (PRISMA-P) checklist. ${ }^{17}$

\section{Eligibility criteria}

Types of studies

All analytical observational epidemiological studies: cohort studies, case control studies and cross-sectional studies on the topic are eligible for inclusion. Animal studies, case series, case studies and reviews will be excluded.

\section{Participants}

We are interested in the role of changes in endogenous sex hormones and in the use of exogenous sex hormones in the year before pregnancy and during pregnancy. We will therefore include women during preconception and pregnancy and their offspring of any age.

\section{Years considered}

We will include studies published from the inception of each database searched until the end of 2016.

\section{Language}

We will have no language restrictions and we will endeavour to translate literature published in languages other than English whenever this is possible.

\section{Information sources}

Database searches and other sources to identify studies

To identify relevant studies, we will search Medline, Embase, Cochrane Library, Institute of Scientific Information (ISI) Web of Science, Cumulative Index of Nursing and Allied Health, Scopus, Google Scholar, Allied and Complementary Medicine Database, Global Health, Psychological Information (PsycINFO), Centre for Agriculture and Bioscience $(\mathrm{CAB})$ International and $\mathrm{WHO}$ Global Health Library. Additional references will be identified by searching the references cited in eligible studies; by searching databases of the proceedings of international conferences, such as ISI Conference Proceedings Citation Index via Web of Science, Zetoc British Library's Electronic Table of Contents (ZETOC) and by contacting a panel of international experts and authors who have published in the field. We will search trial registries, such as Current Controlled Trials (http://www.controlledtrials.com), ClinicalTrials.gov (http://www.clinicaltrials. gov), and Australian and New Zealand Clinical Trials Registry (http://www.anzctr.org.au) to identify ongoing studies.

\section{Search strategy}

We have prepared a search strategy using the Ovid interface for Medline (see online supplementary appendix 1) to identify and retrieve relevant studies. We will adapt this search strategy when searching the other databases.

\section{Study records}

Data management

We will use Endnote Library for managing the references identified from the databases. All records will be exported to Endnote Library for onward study screening, de-duplication and overall management of the retrieved records.

\section{Selection process}

Two reviewers (MT and NM) will independently screen the titles and/or abstracts and full texts of potentially eligible studies. A third reviewer (BN) will arbitrate any disagreements.

\section{Data extraction}

Two reviewers will independently extract relevant study data from eligible studies onto a customised data extraction form, while a third reviewer will arbitrate any discrepancies. The data extraction form will be piloted using a select sample of the eligible studies prior to use in extracting data from all studies.

\section{Data items}

We will produce descriptive summary tables to summarise eligible studies. All relevant study data will be tabulated and as a minimum the tables will include the following: study author, country of study, year of publication, study design, size of study population, source of study population, type of exposure studied (endogenous vs exogenous sex hormones and the specific types) and method of assessment, time before pregnancy and trimester during 
pregnancy exposure was ascertained, length of follow-up (for follow-up studies), confounding factors considered, study outcomes and methods of assessment, analysis methods and key results. The PRISMA checklist will guide the reporting of the systematic review. ${ }^{18}$

\section{Types of exposures}

We will include studies that have investigated the role of all relevant endogenous sex steroid hormones (including oestrogen, progesterone, testosterone) and exogenous sex hormones (including oestrogen only, progesterone only and combined oral contraceptives).

\section{Outcomes and prioritisation}

Our primary outcomes will include: objectively measured or self-reported asthma, atopic dermatitis/eczema, allergic rhinitis, anaphylaxis, urticaria, angioedema and food allergy. The secondary outcomes will include: atopic sensitisation as defined either by skin prick test or raised antigen specific IgE; objective and subjective measures of disease severity and impact on quality of life, including asthma exacerbations, use of asthma medications, hospitalisation for asthma, wheeze as defined by self-report or objective diagnosis; indicators of airway function including (peak expiratory flow, forced expiratory volume in $1 \mathrm{~s}$, forced vital capacity, forced expiratory flow rate or alternative age appropriate pulmonary function tests (oscillometry or exhaled nitric oxide analysis)) and measures of patient-reported health-related quality of life related to asthma or allergy. We will also assess the impact of sex hormones on any allergic or respiratory outcome.

\section{Risk of bias in individual studies}

Two reviewers (MT and NM) will independently evaluate the risk of bias in eligible studies and consensus will be reached through discussion; a third reviewer $(\mathrm{BN})$ will arbitrate any discrepancies. We will use the Effective Public Health Practice Project tool (EPHPP) (www.ephpp. ca) to appraise the risk of bias in studies. The EPHPP tool allows for the grading of key components of the studies, including: suitability of the study design for the research question; risk of selection bias; exposure measurement; outcome assessment (giving a higher grade to objectively defined outcomes than subjectively defined outcomes) and generalisability of findings. From these component-specific gradings, we will derive an overall grading for each study.

\section{Data synthesis}

All analyses will be undertaken separately for studies on exogenous hormones and studies on endogenous hormones. We will synthesise the data both narratively and quantitatively. For quantitative synthesis, we will perform random-effects meta-analysis to combine the estimates from studies judged to be clinically, methodologically and statistically homogeneous. Heterogeneity between studies will be quantified using the $\mathrm{I}^{2}$ statistic. For analyses investigating the role of endogenous sex hormones, data syntheses will be undertaken by pregnancy trimester.
Subgroup analyses will be performed according to age of offspring at onset/diagnosis of outcomes (where possible using the following age groups: $<5$ years, $5-12$ years, $>12$ years), sex, parity and for analyses investigating the role of exogenous sex hormones, time of exposure before pregnancy (eg, 6 months vs 12 months before pregnancy). Using the grading derived from the quality appraisal of eligible studies, we will perform sensitivity analyses to evaluate the robustness of our findings and whether they are influenced by the risk of bias in studies. The meta-analyses will be performed using the statistical package Stata V.14.

\section{Publication bias}

We will evaluate the potential for publication bias by using funnel plots and Begg and Egger tests. ${ }^{19} 20$

\section{Confidence in the cumulative estimate}

We will evaluate and grade the strength and quality of the overall evidence emanating from the review using the Grading of Recommendations Assessment, Development and Evaluation approach. ${ }^{21}$

We do not envisage making clinical recommendations from this largely and exclusively epidemiological evidence base. There may be no need to interfere with pregnancy hormones in otherwise healthy females. However, if prenatal endogenous hormones contribute to the risk of allergy and asthma in the offspring, understanding this role and the mechanisms through which these hormones operate is essential for the field, as this will contribute to knowledge of the fetal origin of allergy and asthma. Moreover, since studies have now reported such associations, with varying results, a synthesis of the underlying evidence is essential in clarifying any putative role of prenatal sex hormones in the development of asthma and allergy in the offspring.

\section{ETHICS AND DISSEMINATION}

We completed the Usher Institute of Population Health Sciences and Informatics Level 1 ethics self-assessment form, which indicated that no further ethical permission was required for this work as the study will only involve review of the published literature. Our findings will be reported in a peer-reviewed scientific journal.

\section{Author affiliations}

${ }^{1}$ Asthma UK Centre for Applied Research, Centre for Medical Informatics, Usher Institute of Population Health Sciences and Informatics, The University of Edinburgh, Edinburgh, UK

${ }^{2}$ Department of Food and Environmental Sciences, University of Helsinki, Helsinki, Finland

${ }^{3}$ Department of Pediatrics, Public Health Medicine, University of Helsinki and Helsinki University Hospital, University of Helsinki, Tampere University Hospital, Helsinki, Finland

${ }^{4}$ School of Health Sciences, University of Tampere, Tampere, Finland

${ }^{5}$ Department of Lifestyle and Participation, Nutrition Unit, National Institute for Health and Welfare, Helsinki, Finland

${ }^{6}$ Tampere Centre for Child Health Research, Tampere University Hospital, Tampere, Finland 
${ }^{7}$ Science Centre of Pirkanmaa Hospital District, Tampere University Hospital and University of Tampere, Tampere, Finland

Contributors BN conceived the idea for this work and is the guarantor. AS contributed subject expertise to the development of the protocol. The protocol was drafted by MT and BN and was then revised after several rounds of critical comments from AS and additional feedback from MK, ME, SV and NM. All authors are involved in the systematic review process.

Funding MT undertakes this work as part of the requirement for her dissertation for the award of a Master's in Public Health at The University of Edinburgh. The work received no specific funding. BN was supported by the Institute for Advanced Social Research Fellowship, University of Tampere, Finland; School of Health Sciences, University of Tampere, Finland. Additional support was received from the Farr Institute and Asthma UK Centre for Applied Research. The views presented here are those of the authors and not necessarily those of the Universities of Tampere and Edinburgh.

Competing interests None declared.

Ethics approval Level 1 ethics form from Edinburgh Institutional Review Board was completed for this study, which indicated that no ethics approval is required since it is based only on the published literature.

Provenance and peer review Not commissioned; externally peer reviewed.

Open Access This is an Open Access article distributed in accordance with the Creative Commons Attribution Non Commercial (CC BY-NC 4.0) license, which permits others to distribute, remix, adapt, build upon this work non-commercially, and license their derivative works on different terms, provided the original work is properly cited and the use is non-commercial. See: http://creativecommons.org/ licenses/by-nc/4.0/

C Article author(s) (or their employer(s) unless otherwise stated in the text of the article) 2017. All rights reserved. No commercial use is permitted unless otherwise expressly granted.

\section{REFERENCES}

1. Costantine MM. Physiologic and pharmacokinetic changes in pregnancy. Front Pharmacol 2014;5:65

2. Thornburg KL, Jacobson SL, Giraud GD, et al. Hemodynamic changes in pregnancy. Semin Perinatol 2000;24:11-14.

3. Chesnutt AN. Physiology of normal pregnancy. Crit Care Clin 2004;20:609-15.
4. Schindler AE. First trimester endocrinology: consequences for diagnosis and treatment of pregnancy failure. Gynecol Endocrinol 2004;18:51-7.

5. Newbern D, Freemark M. Placental hormones and the control of maternal metabolism and fetal growth. Curr Opin Endocrinol Diabetes Obes 2011;18:409-16.

6. Pincus M, Keil T, Rücke M, et al. Fetal origin of atopic dermatitis. J Allergy Clin Immunol 2010;125:273-5.

7. Xu B, Pekkanen J, Husman T, et al. Maternal sex hormones in early pregnancy and asthma among offspring: a case-control study. $J$ Allergy Clin Immunol 2003;112:1101-4.

8. Shaheen SO, Hines M, Newson RB, et al. Maternal testosterone in pregnancy and atopic outcomes in childhood. Allergy 2007;62:25-32.

9. Hartwig IR, Bruenahl CA, Ramisch K, et al. Reduced levels of maternal progesterone during pregnancy increase the risk for allergic airway diseases in females only. J Mol Med 2014;92:1093-104.

10. Wjst M, Dold S. Is asthma an endocrine disease? Pediatr Allergy Immunol 1997:8:200-4.

11. Yamamoto-Hanada K, Futamura M, Yang L, et al. Preconceptional exposure to oral contraceptive pills and the risk of wheeze, asthma and rhinitis in children. Allergol Int. In Press. 2016;65.

12. Brooks K, Samms-Vaughan M, Karmaus W. Are oral contraceptive use and pregnancy complications risk factors for atopic disorders among offspring? Pediatr Allergy Immunol 2004;15:487-96.

13. Frye C, Mueller JE, Niedermeier K, et al. Maternal oral contraceptive use and atopic diseases in the offspring. Allergy 2003;58:229-32.

14. Hancock DB, Håberg SE, Furu K, et al. Oral contraceptive pill use before pregnancy and respiratory outcomes in early childhood. Pediatr Allergy Immunol 2011;22:528-36.

15. Keski-Nisula L, Pekkanen J, Xu B, et al. Does the pill make a difference? Previous maternal use of contraceptive pills and allergic diseases among offspring. Allergy 2006;61:1467-72.

16. Xu B, Järvelin MR, Pekkanen J. Prenatal factors and occurrence of rhinitis and eczema among offspring. Allergy 1999;54:829-36.

17. Shamseer L, Moher D, Clarke M, et al. Preferred reporting items for systematic review and meta-analysis protocols (PRISMA-P) 2015: elaboration and explanation. BMJ 2015;349:g7647.

18. Moher D, Liberati A, Tetzlaff J, et al. Preferred reporting items for systematic reviews and meta-analyses: the PRISMA statement. PLoS Med 2009;6:e1000097.

19. Begg CB, Mazumdar M. Operating characteristics of a rank correlation test for publication bias. Biometrics 1994;50:1088-101.

20. Egger M, Davey Smith G, Schneider M, et al. Bias in meta-analysis detected by a simple, graphical test. BMJ 1997;315:629-34.

21. Guyatt GH, Oxman AD, Vist GE, et al. GRADE: an emerging consensus on rating quality of evidence and strength of recommendations. BMJ 2008;336:924-6. 